\title{
Constraints on Dark Matter with Gamma-Ray Experiments and Future Observational Strategies
}

\author{
Aldo Morselli * \\ INFN, Rome Tor Vergata, Rome, Italy
}

Indirect dark matter (DM) searches rely on detection of stable by-products of DM interactions to search for a signal of this elusive component of the Universe. Among these final products, gamma rays have recently played a major role in understanding the nature of the DM particle. We review the current status of indirect DM searches with gamma-ray observations and prospects with future instruments.

Keywords: gamma rays, dark matter, detectors in space, astroparticle physics

\section{INTRODUCTION}

OPEN ACCESS

Edited by:

Nicolao Fornengo,

University of Turin, Italy

Reviewed by:

Marco Cirelli,

Centre National de la Recherche

Scientifique (CNRS), France

Chiara Arina

Institut d'astrophysique de Paris,

France

*Correspondence:

Aldo Morselli

aldo.morselli@roma2.infn.it

Specialty section:

This article was submitted to

High-Energy and Astroparticle

Physics,

a section of the journal

Frontiers in Physics

Received: 20 September 2016

Accepted: 17 January 2017

Published: 14 February 2017

Citation:

Morselli A (2017) Constraints on Dark Matter with Gamma-Ray Experiments and Future Observational Strategies.

doi: 10.3389/fphy.2017.00003
High-energy phenomena in the cosmos, and in particular processes leading to the emission of gamma- rays in the energy range $300 \mathrm{KeV}-100 \mathrm{TeV}$, play a very special role in the understanding of our Universe. This energy range is indeed associated with non-thermal phenomena and challenging particle acceleration processes. The Universe can be thought as a context where fundamental physics, relativistic processes, strong gravity regimes, and plasma instabilities can be explored in a way that is not possible to reproduce in our laboratories. High-energy astrophysics and atmospheric plasma physics are indeed not esoteric subjects, but are strongly linked with our daily life. Understanding cosmic high-energy processes has a large impact on our theories and laboratories applications. The technology involved in detecting gamma-rays is challenging and drives our ability to develop improved instruments for a large variety of applications.

The energy range between $300 \mathrm{Kev}$ and $100 \mathrm{MeV}$ is an experimentally very difficult range and remained uncovered since the time of COMPTEL. In this range a new instrument can address all astrophysics issues left open by the current generation of instruments. In particular better angular resolution in the energy range $10 \mathrm{MeV}-1 \mathrm{GeV}$ is crucial to resolve patchy and complex features of diffuse sources in the Galaxy and in the Galactic Centre as well as increasing the point source sensitivity. This instrument can address scientific topics of great interest to the community, with particular emphasis on multifrequency correlation studies involving radio, optical, IR, X-ray, soft gamma-ray and $\mathrm{TeV}$ emission.

Above $100 \mathrm{MeV}$, thanks to the launch of the Fermi-LAT satellite and to the advent of a new generation of imaging air Cherenkov telescopes (H.E.S.S., MAGIC, VERITAS), several thousand gamma-ray sources are known today revealing an unexpected ubiquity of particle acceleration processes in the Universe.

Major scientific challenges are still ahead, such as the identification of the nature of dark matter, the discovery and understanding of the sources of cosmic rays, or the comprehension of the particle acceleration processes that are at work in the various objects.

The identification of the nature of dark matter can be done with the detection of gamma rays and cosmic rays from the annihilation or decay of dark matter particles. This is a promising method for identifying dark matter, understanding its intrinsic properties, and mapping its distribution in the universe. 


\subsection{Search for Dark Matter in the Galactic Center and in the Dwarf Spheroidal Galaxy Satellites}

Astrophysical searches for dark matter are a fundamental part of the experimental efforts to explore the dark sector. The strategy is to search for DM annihilation products in preferred regions of the sky, i.e., those with the highest expected DM concentrations and still close enough to yield high DM-induced fluxes at the Earth. For that reason, the Galactic Center (GC), nearby dwarf spheroidal galaxy (dSphs) satellites of the Milky Way, as well as local galaxy clusters are thought to be among the most promising objects for DM searches. In particular, dSphs represent very attractive targets because they are highly DM-dominated systems and are expected to be free from any other astrophysical gammaray emitters that might contaminate any potential DM signal. Although the expected signal cannot be as large as that from the GC, dSphs may produce a larger signal-to-noise $(S / N)$ ratio. This fact allows us to place very competitive upper limits on the gamma-ray signal from DM annihilation [1-3], using data collected by the Large Area Telescope (LAT) onboard the Fermi gamma-ray observatory [4]. These are often referred to as the most stringent limits on DM annihilation cross-section obtained so far.

Despite these interesting limits derived from dSphs, the GC is still expected to be the brightest source of DM annihilations in the gamma-ray sky by several orders of magnitude. Although several astrophysical processes at work in the crowded GC region make it extremely difficult to disentangle the DM signal from conventional emissions, the DM-induced gamma-ray emission is expected to be so large there that the search is still worthwhile. Furthermore, the DM density in the GC may be larger than what is typically obtained in $\mathrm{N}$-body cosmological simulations. Ordinary matter (baryons) dominates the central region of our Galaxy [5]. Thus, baryons may significantly affect the DM distribution. As baryons collapse and move to the center they increase the gravitational potential, which in turn forces the
DM to contract and increase its density. This is a known and qualitatively well understood physical process [6]. It is also observed in many cosmological simulations that include hydrodynamics and star formation [7]. If this is the only effect of baryons, then the expected annihilation signal will substantially increase $[5,8]$ but another possible effect of baryonic matter is that of blastwave supernova feedback, that has the opposite impact on the DM density profile [9].

A preliminary analysis of Fermi LAT observations of the GC region was presented in Vitale [10] Morselli et al. [11]. with an observation of an excess of gamma rays in the 3-5 GeV energy range from the GC region. These results produced a lot of activity outside the Fermi collaboration with claims of evidence for dark matter in the Galactic Center (i.e., [12], [13] and references therein).

This possibility was already considered in the analysis of the EGRET galactic center excess [14] but there are other possible explanations, e.g., a population of millisecond pulsars around the Galactic Center below the Fermi threshold $[15,16]$.

A third possibility is related to past activity of the Galactic Center $[17,18]$. In this case the excess can be connected to the Fermi bubble and it will be very important to see how this bubble is structured in the GC region.

Another possibility is related to extra injections of cosmic rays (steady-state, not related to the past activity of the GC), which produce radiation. See for example [19-21].

The analysis of the Fermi Collaboration [22] using 5 years of data and the Pass 7 event selections [23] and the on-going analysis with 6.5 year of data and the Pass 8 event selections [24] confirm the excess but confirm also that when all the uncertainties on the excess morphology and spectrum related to the modeling of the various components of gamma-ray emission in that region, in particular in the distribution of interstellar gas along the line of sight, in the low latitude emission from the Fermi bubbles, and in the abundance of cosmic ray sources in the innermost Galaxy are considered the spectrum varies significantly and it is not possible
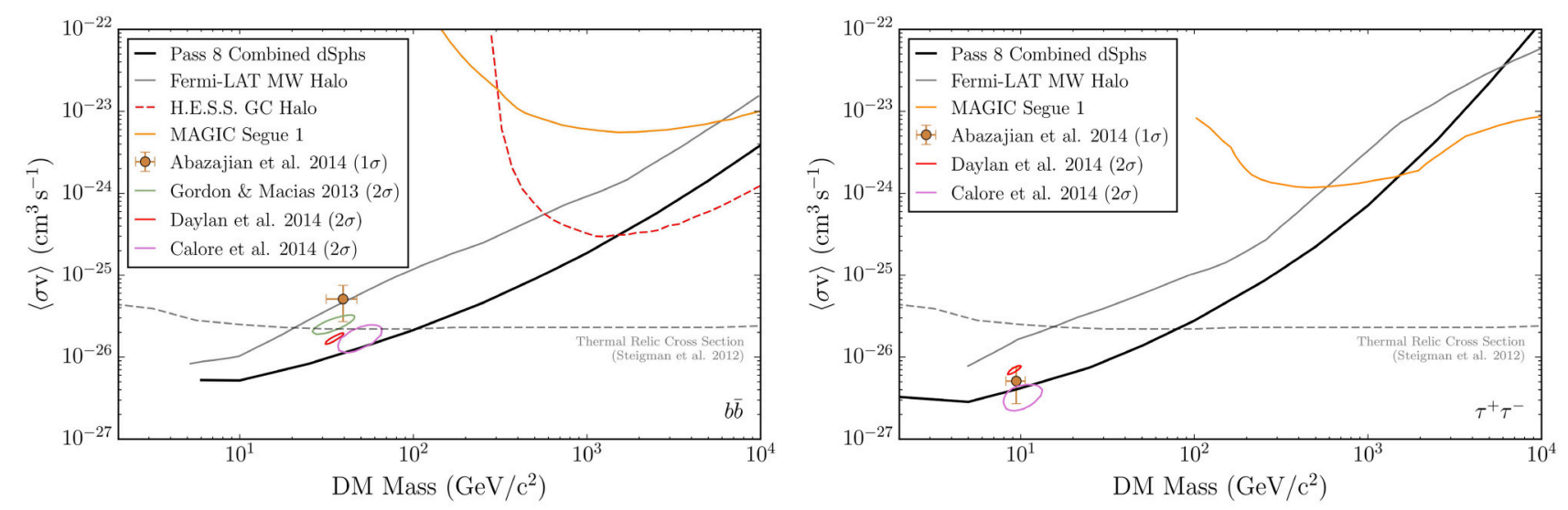

FIGURE 1 | Comparison of constraints on the DM annihilation cross section for the $\bar{b} \boldsymbol{b}$ (Left) and $\bar{\tau} \tau$ (Right) channels [25] with previously published constraints from LAT analysis of the Milky Way halo (3 $\sigma$ limit) [26], $112 \mathrm{~h}$ of observations of the Galactic Center with H.E.S.S. [27], and 157.9 h of observations of Segue 1 with MAGIC [28]. These limits have been found to be fairly insensitive to the assumed DM density profile [25]. Closed contours and the marker with error bars show the best-fit cross section and mass from several interpretations of the Galactic center excess [12]. 


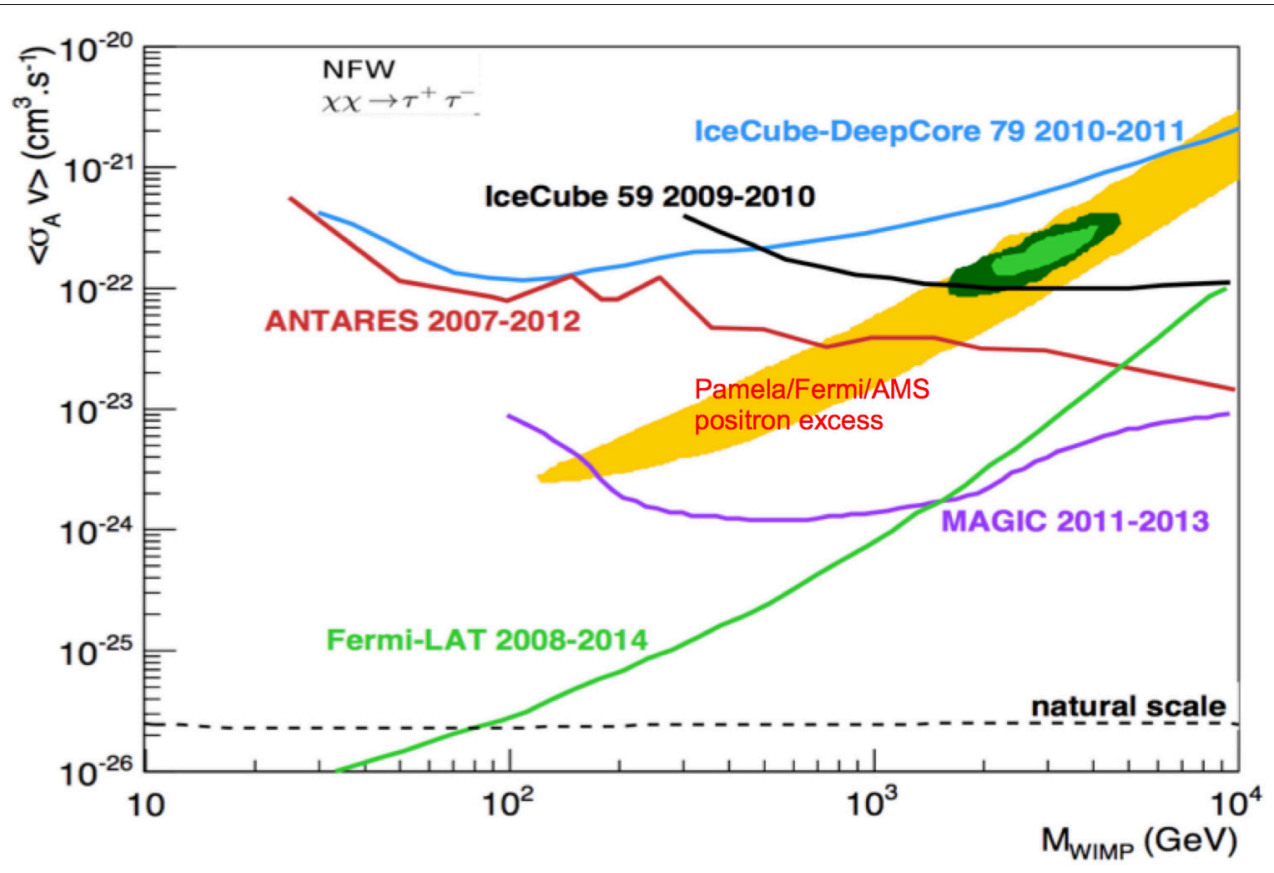

FIGURE 2 | Comparison of Fermi-LAT constraints on the DM annihilation cross section for the $\bar{\tau} \tau$ channel [25] with Antares [29], IceCube-DeepCore [30] and MAGIC [28].

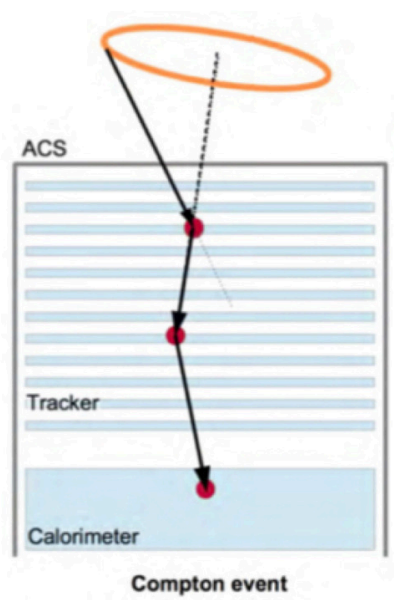

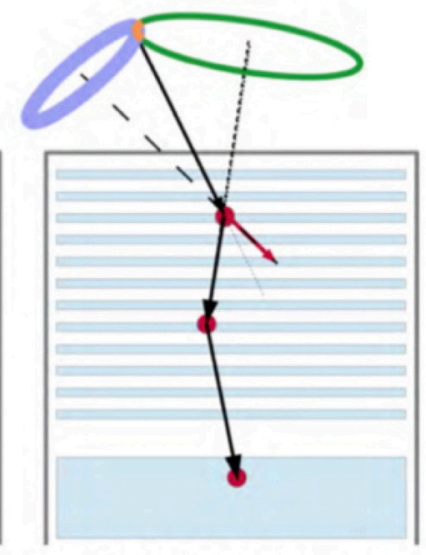

Tracked Compton event

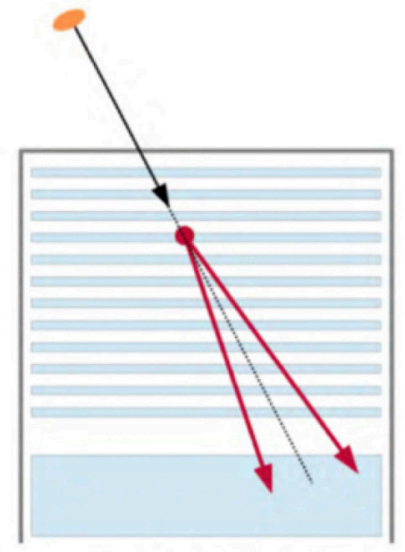

Pair event

FIGURE 3 | Representative event topologies for Compton events without (Left) and with electron tracking (Center) and for a pair event (Right) inside the e-ASTROGAM detector.

to discriminate between the different hypotesis. The new analysis of the dSphs with the use of Pass 8 begin to constrain some of the preferred parameter space for a DM interpretation of a gammaray excess in the Galactic center region. As shown in Figure 1, for interpretations assuming a $\bar{b} b$ final state, the best-fit models lie in a region of parameter space slightly above the 95\% CL upper limit from this analysis, with an annihilation cross section in the range of $(1-3) \times 10^{-26} \mathrm{~cm}^{3} \mathrm{~s}^{-1}$ and $m_{D M}$ between 25 and 50 $\mathrm{GeV}$. However, uncertainties in the structure of the Galactic DM distribution can significantly enlarge the best-fit regions of $\langle\sigma v\rangle$ channel, and $m_{D M}$.
Figure 2 shows a comparison of constraints on the DM annihilation cross section for the $\bar{\tau} \tau$ channel [25] with Antares [29], IceCube-DeepCore [30] and MAGIC [28]. One can see that the Fermi limits are the best limits below $2 \mathrm{TeV}$.

At lower energies a new instrument like Gamma-Light [31], or ASTROGAM can really improve these results both in the Galactic center and in the dSphs limits.

The project for an improved version of ASTROGAM, eASTROGAM, is being prepared in reply of the fifth ESA call for medium mission ( M5 ) that will be released in the fall of 2016. $[32,33]$. 
Interactions of photons with matter in the e-ASTROGAM energy range is dominated by Compton scattering from 0.1 $\mathrm{MeV}$ up to about $15 \mathrm{MeV}$ in silicon, and by electron-positron pair production in the field of a target nucleus at higher energies. e-ASTROGAM maximizes its efficiency for imaging and spectroscopy of energetic gamma rays by using both processes. The e-ASTROGAM instrument is based on doublesided Silicon detectors coupled to front-end-electronics capable of acquiring analog information on energy deposition in the range 20-1000 keV with high efficiency and high signalto-noise. Both Compton events induced by photons in the range $0.3-30 \mathrm{MeV}$ and pair production events in the $30 \mathrm{MeV}-$ $30 \mathrm{GeV}$ range can be detected by the e-ASTROGAM Tracker equipped with a Calorimeter and an Anticoincidence system.

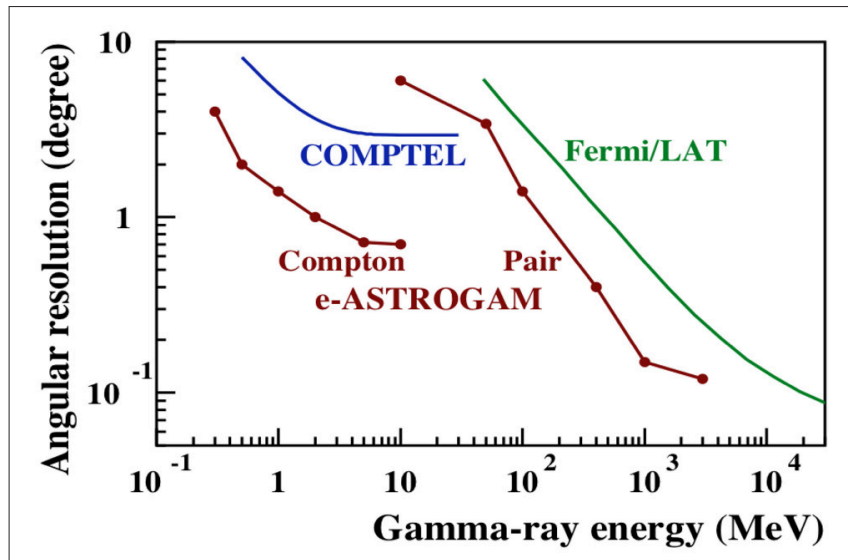

FIGURE 4 | Point Spread Function (PSF, 68\% containment radius) of the e-ASTROGAM gamma-ray detector. For comparison, we show the Fermi-LAT Pass7 PSF and the COMPTEL instrument. In the Compton domain, the performance of e-ASTROGAM and COMPTEL is the FWHM of the angular resolution measure (ARM) .
Figure 3 shows representative topologies for Compton and pair events.

For Compton events, point interactions of the gamma ray in tracker and calorimeter produce spatially resolved energy deposits, which have to be reconstructed in sequence using the redundant kinematic information from multiple interactions. Once the sequence is established, two sets of information are used for imaging: the total energy and the energy deposit in the first interaction measure the first Compton scatter angle. The combination with the direction of the scattered photon from the vertices of the first and second interactions generates a ring on the sky containing the source direction. Multiple photons from the same source enable a full deconvolution of the image, using probabilistic techniques. For energetic Compton scatters (above $1 \mathrm{MeV}$ ), measurement of the track of the scattered electron becomes possible, resulting in a reduction of the event ring to an arc, hence further improving event reconstruction. Compton scattering depends on polarization of the incoming photon, hence careful statistical analysis of the photons for a strong (e.g., transient) source yields a measurement of the degree of polarization of its high-energy emission. Pair events produce two main tracks from the electron and positron at small opening angle. Tracking of the initial opening angle and the plane spanned by electron and positron enables direct back-projection of the source. Multiple scattering in the tracker material (or any intervening passive materials) leads to broadening of the tracks and limits the angular resolution at low energies.

The nuclear recoil taking up an unmeasured momentum results in a small uncertainty, usually negligible compared to instrumental effects. The energy of the gamma ray is measured using the calorimeter. Polarization information in the pair domain is given by the azimuthal orientation of the electron-positron plane. The Point Spread Function of

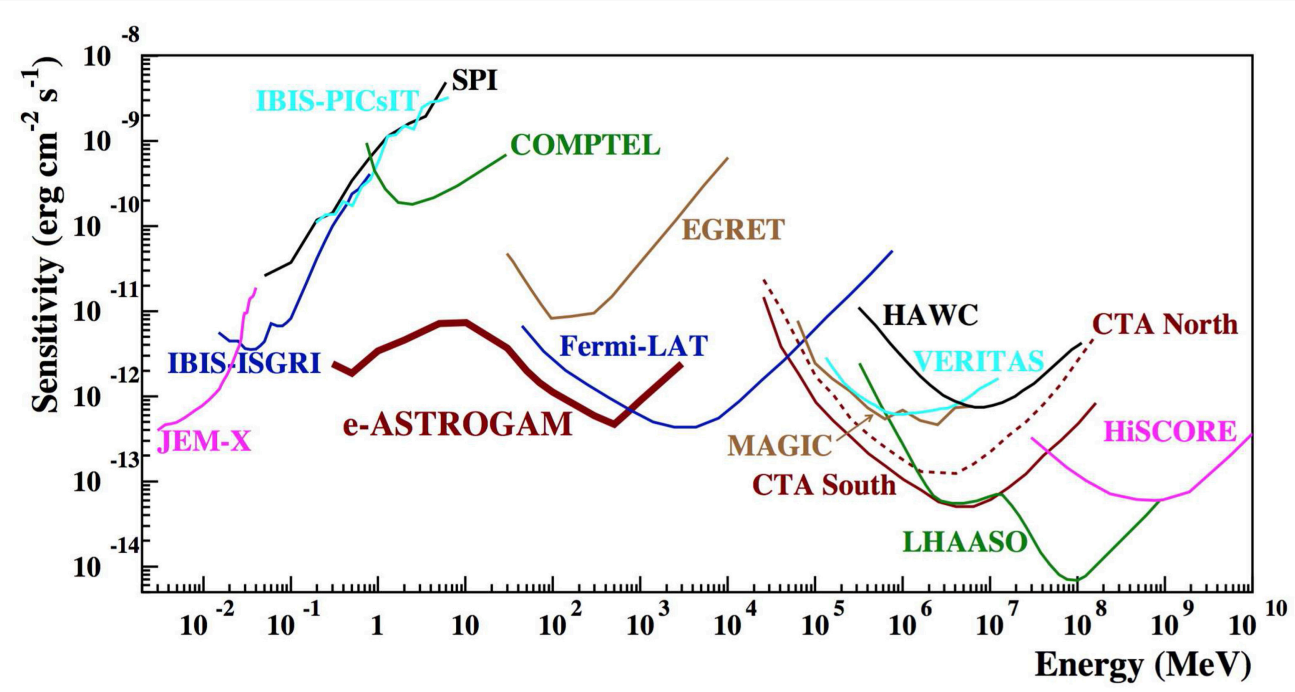

FIGURE 5 | Point source continuum sensitivity of different $X$ and $\gamma$-ray instruments compared with e-ASTROGAM. 

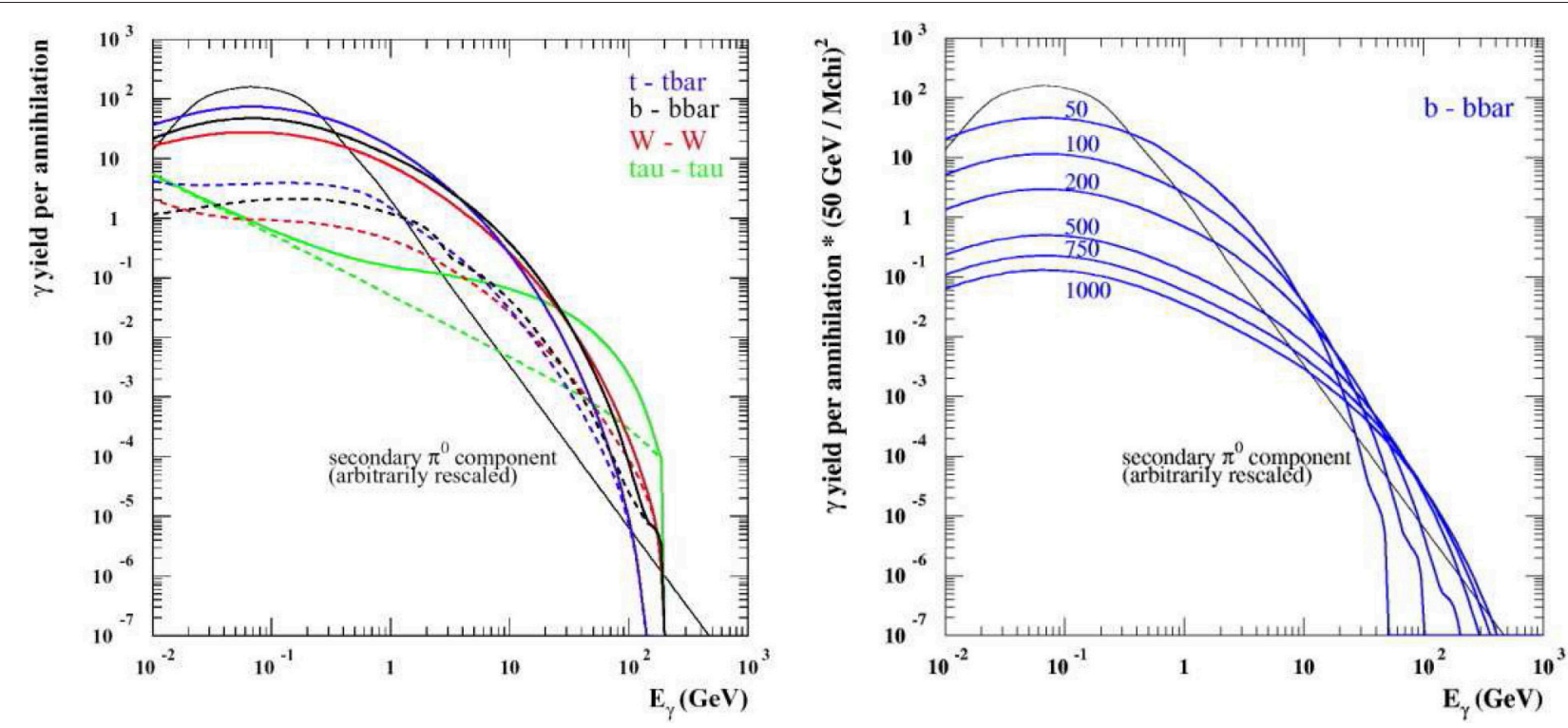

FIGURE 6 | (Left) Differential energy spectra per annihilation for a few sample annihilation channels and a fixed WIMP mass (200 GeV) and differential $\gamma$-ray energy spectra per annihilation for a fixed annihilation channel (bb) and for different values of WIMP masses [14]. For comparison we also show the emissivity, with an arbitrarily rescaled normalization, from the interaction of primaries with the interstellar medium. (Right) The solid lines are the total yields for different annihilation channels, while the dashed lines are components not due to $\pi^{0}$ decays.

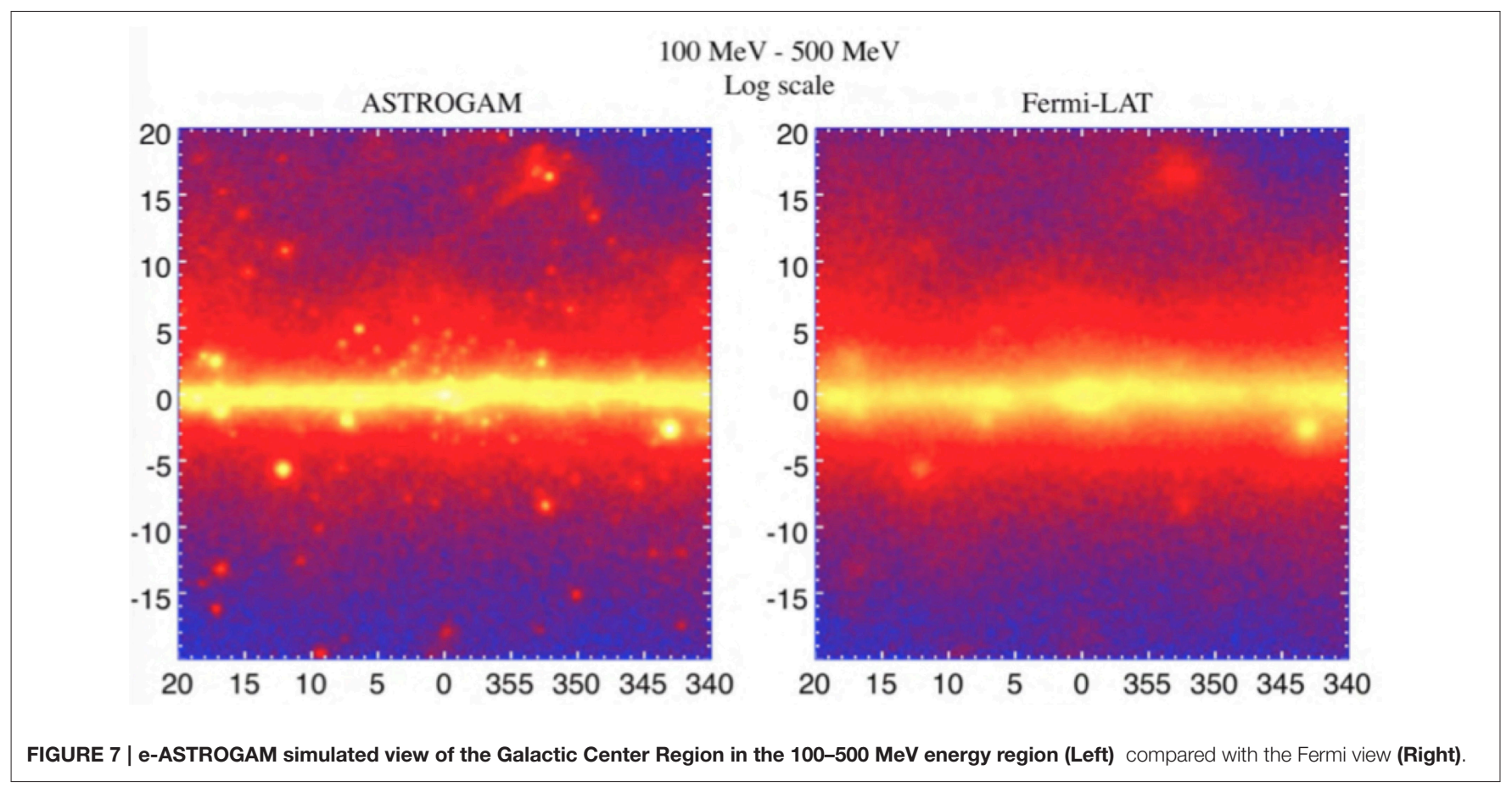

e-ASTROGAM is shown in Figure 4 , and the sensitivity is shown in Figure 5 for an effective exposure of 1 year of a high Galactic latitude source. Sensitivities above 30 $\mathrm{MeV}$ are given at the 5-sigma confidence level, whereas those below $10 \mathrm{MeV}$ (30 MeV for COMPTEL) are at 3sigma.

\section{DARK MATTER STUDIES IN THE MEV-GEV DOMAIN}

One of the major scientific objectives of e-ASTROGAM is the search for dark matter by means of the production of secondary gamma-rays after the annihilation or decay of the DM particle 
candidates. The importance of e-ASTROGAM for DM searches can be seen in Figure 6 where the differential $\gamma$-ray energy spectra per annihilation of WIMP are plotted [14]. As one can see the bulk of the emission even for high WIMP masses is in the energy range 5-100 $\mathrm{MeV}$. Decaying DM can also produce a detectable line in the e-ASTROGAM energy range that might be detectable out of the continuum. Together with Fermi and CTA, e-ASTROGAM will probe most of the space of WIMP models with thermal relic annihilation cross section.

Resolving the inner region of our Galaxy at high-energies remains one of the outstanding problems of modern astrophysics. Despite several attempts, the origin of positrons currently annihilating at the rate of $2 \cdot 10^{43} \mathrm{~s}^{-1}$ from the inner Galaxy is not accounted for by current models of star formation and compact object activities in the region. Recent data show that in addition to the central bulge also the inner disk is producing $511 \mathrm{keV}$ emission. Candidate positron sources include: the central black hole activity, massive stars, Supernovae, compact binaries, pulsars, and possibly DM annihilation/deexcitation. The much improved e-ASTROGAM sensitivity at the electron-positron annihilation energy will be used for a high-resolution mapping of the mysterious $511 \mathrm{KeV}$ radiation. In the Fermi-LAT analysis of the Galactic Center the diffuse

\section{REFERENCES}

1. Ackermann M, Ajello M, Albert A, Atwood WB, Baldini L, Ballet J, et al. Constraining dark matter models from a combined analysis of milky way satellites with the fermi large area telescope. Phys Rev Lett. (2011) 107:241302. doi: 10.1103/PhysRevLett.107.241302

2. Abdo AA, Ackermann M, Ajello M, Atwood WB, Baldini L, Ballet J, et al. Observations of milky way dwarf spheroidal galaxies with the fermiLAT detector and constraints on dark matter models. Astrophys J. (2010) 712:147-58. doi: 10.1088/0004-637X/712/1/147

3. Ackermann M, Albert A, Anderson B, Atwood WB, Baldini L, Barbiellini G, et al. Constraining dark matter models from a combined analysis of milky way satellites with the fermi large area telescope. Phys Rev Lett. (2015) 115:231301. doi: 10.1103/PhysRevLett.115.231301

4. Atwood WB, Abdo AA, Ackermann M, Anderson B, Axelsson M, Baldini $\mathrm{L}$, et al. The large area telescope on the fermi gamma-ray space telescope mission. Astrophys J. (2009) 697:1071. doi: 10.1088/0004-637X/697/2/ 1071

5. Prada F, Klypin A, Flix Molina J, Martinez M, Simonneau E. Dark matter annihilation in the milky way galaxy: effects of baryonic compression. Phys Rev Lett. (2004) 93:241301. doi: 10.1103/PhysRevLett.93.241301

6. Zeldovich YaB, Klypin AA, Khlopov YuM, Chechetkin VM. Astrophysical constraints on the mass of heavy stable neutral leptons. Sov J Nucl Phys. (1980) 31:664.

7. Gustafsson M, Fairbairn M, Sommer-Larsen J. Baryonic pinching of galactic dark matter halos. Phys Rev D (2006) 74:123522. doi: 10.1103/PhysRevD.74.123522

8. Mambrini Y, Muñoz C, Nezri E, Prada F. Adiabatic compression and indirect detection of supersymmetric dark matter. JCAP (2006) 0601:010. doi: 10.1088/1475-7516/2006/01/010

9. Di Cintio A, Brook CB, Maccio AV, Stinson GS, Knebe A, Dutton AA, et al. MNRAS (2013) 437:415-23. arXiv:1306.0898v2.

10. Vitale V, Morselli A. Indirect search for dark matter from the center of the milky way with the fermi-large area telescope. In: 2009 Fermi Symposium, eConf Proceedings C091122 Washington, DC.

11. Morselli A, Cañadas, B, Vitale V. The indirect search for dark matter from the centre of the galaxy with the fermi LAT. gamma-ray backgrounds and discrete sources, as we model them today, can account for the large majority of the detected gamma-ray emission from the Galactic Center.

Nevertheless, a residual emission is left, not accounted for by the above models of standard astrophysical phenomena. In the crowded Galactic Center region the analysis to disentangle a possible DM signal from conventional emissions has still large uncertainties due to the extremely difficult subtraction of the Galactic diffuse emission and the contribution of unresolved sources. The very good angular resolution of e-ASTROGAM at low energies will help to resolve sources in the galactic center region and to disentangle the possible DM contribution, see Figure 7.

e-ASTROGAM will also perform indirect DM detection searches in dwarf spheroidal galaxies and put constraints on DM contribution to the largely unknown diffuse extragalactic gamma-ray background in the spectral range $0.3-100 \mathrm{MeV}$. Models will be tested in a spectral range not yet currently studied.

\section{AUTHOR CONTRIBUTIONS}

The author confirms being the sole contributor of this work and approved it for publication.
Il Nuovo Cimento (2011) 34:311-6. doi: 10.1393/ncc/i201110896-7

12. Calore F, Cholis I, Weniger C. Background model systematics for the Fermi GeV excess. JCAP 03 (2015) 038:1503-38. doi: $10.1088 / 1475-7516 / 2015 / 03 / 038$

13. Daylan T, Finkbeiner DP, Hooper D, Linden T, Portillo SKN, Rodd NL, et al. The characterization of the gamma-ray signal from the central milky way: a compelling case for annihilating dark matter. Phys Dark Univ. (2016) 12:123. doi: 10.1016/j.dark.2015.12.005

14. Cesarini A, Fucito F, Lionetto A, Morselli A, Ullio P. The galactic center as a dark matter gamma-ray source. Astropart Phys. (2004) 21:267. doi: 10.1016/j.astropartphys.2004.02.001

15. Lee SK, Lisanti M, Safdi BR, Slatyer TR, Xue W. Evidence for unresolved gamma-ray point sources in the inner galaxy. Phys Rev Lett. (2016) 116:051103. doi: 10.1103/PhysRevLett.116.051103

16. Bartels R, Krishnamurthy S, Weniger C. Strong support for the millisecond pulsar origin of the galactic center $\mathrm{GeV}$ excess. Phys Rev Lett. (2016) 116:051102. doi: 10.1103/PhysRevLett.116. 051102

17. Petrovic J, Serpico PD, Zaharijas G. Galactic center gamma-ray "excess" from an active past of the galactic centre? JCAP (2014) 10:052. doi: 10.1088/1475-7516/2014/10/052

18. Carlson E, Profumo S. Cosmic ray protons in the inner Galaxy and the Galactic Center gamma-ray excess. Phys Rev D (2014) 90:023015. doi: 10.1103/PhysRevD.90.023015

19. Gaggero D, Taoso M, Urbano A, Valli M, and Ullio P. Towards a realistic astrophysical interpretation of the gamma-ray Galactic center excess. JCAP (2015) 12:056. doi: 10.1088/1475-7516/2015/12/056

20. Carlson E, Profumo S, Linden T. Putting things back where they belong: tracing cosmic-ray injection with H2. Phys Rev Lett. (2016) 117:111101. doi: 10.1103/PhysRevLett.117.111101

21. Carlson E, Linden T, Profumo S. Improved cosmic-ray injection models and the galactic center gamma-ray excess. Phys Rev D (2016) 94:063504. doi: 10.1103/PhysRevD.94.063504

22. Ajello M, Albert A, Atwood WB, Barbiellini G, Bastieri D, Bechtol K, et al. "Fermi-LAT observations of high-energy gamma-ray emission toward the galactic center. Astrophys J. (2016) 819:44. doi: 10.3847/0004-637X/819/1/44 
23. Ackermann M, Ajello M, Albert A, Allafort A, Atwood WB, Axelsson $\mathrm{M}$, et al. The fermi large area telescope on orbit: event classification, instrument response functions, and calibration. Astrophys J Suppl. (2012) 203:4. doi: 10.1088/0067-0049/203/1/4

24. Ackermann M, Ajello M, Albert A, Anderson B, Atwood WB, Baldini L, et al. Updated search for spectral lines from galactic dark matter interactions with pass 8 data from the fermi large area telescope. Phys Rev D (2015) 91:122002. doi: 10.1103/PhysRevD.91.122002

25. Ackermann M, Albert A, Anderson B, Atwood WB, Baldini L, Barbiellini G, et al. Searching for dark matter annihilation from milky way dwarf spheroidal galaxies with six years of fermi-LAT data. Phys Rev Lett. (2015) 115:231301. doi: 10.1103/PhysRevLett.115.231301

26. Ackermann M, Ajello M, Atwood WB, Baldini L, Barbiellini G, Bastieri D, et al. Constraints on the galactic halo dark matter from fermi-LAT diffuse measurements. Astrophys J. (2012) 761:91. doi: 10.1088/0004-637X/761/2/91

27. Abramowski A, Acero F, Aharonian F, Akhperjanian AG, Anton G, Barnacka A, et al. Search for a dark matter annihilation signal from the galactic center halo with H.E.S.S. Phys Rev Lett. (2011) 106:161301. doi: 10.1103/PhysRevLett.106.161301

28. Aleksić J, Ansoldi S, Antonelli LA, Antoranz P, Babic A, Bangale $\mathrm{P}$, et al. Optimized dark matter searches in deep observations of Segue 1 with MAGIC. J Cosmol Astropart Phys. (2014) 1402:008. doi: 10.1088/1475-7516/2014/02/008

29. Adrían-Martínez S, Albert A, Andr M, Anton G, Ardid M, Aubert JJ, et al. Search of dark matter annihilation in the galactic centre using the ANTARES Neutrino Telescope (2015) arXiv:1505.04866.
30. Aartsen MG, Abbasi R, Abdou Y, Ackermann M, Adams J, Aguilar JA, et al The IceCube neutrino observatory part IV: searches for dark matter and exotic particles (2013) arXiv:1309.7007.

31. Morselli A, Argan A, Barbiellini G, Bonvicini W, Bulgarelli A, Cardillo $\mathrm{M}$, et al. "Gamma-light: high-energy astrophysics above $10 \mathrm{MeV}$. Nucl Phys B (2013) 239-40:193-8. doi: 10.1016/j.nuclphysbps.2013. 05.030

32. Tatischeff V, Tavani M, von Ballmoos P, Hanlon L, Oberlack U, Aboudan A, et al. The e-ASTROGAM gamma-ray space mission. In: Proc. SPIE 9905, Space Telescopes and Instrumentation 2016: Ultraviolet to Gamma Ray, 99052N Edinburgh.

33. De Angelis A, Tatischeff V, Tavani M, Oberlack U, Grenier I, Hanlon $\mathrm{L}$, et al. The e-ASTROGAM mission (exploring the extreme Universe with gamma rays in the $\mathrm{MeV}-\mathrm{GeV}$ range). Exp Astron. (2016) arXiv:1611. 02232 .

Conflict of Interest Statement: The author declares that the research was conducted in the absence of any commercial or financial relationships that could be construed as a potential conflict of interest.

Copyright (C) 2017 Morselli. This is an open-access article distributed under the terms of the Creative Commons Attribution License (CC BY). The use, distribution or reproduction in other forums is permitted, provided the original author (s) or licensor are credited and that the original publication in this journal is cited, in accordance with accepted academic practice. No use, distribution or reproduction is permitted which does not comply with these terms. 